(Aus dem Pathologisch-anatomischen Institut der Stadt Magdeburg [Prof. Dr. Ricker].)

\title{
Gibt es eine kongenitale örtliche Disposition zur Bildung otosklerotischer Knochenherde?
}

Von

Dr. W. Schoetz, Berlin.

(Mit Tafel XXVII/XXIX).

Die anatomischen Veränderungen am Felsenbein, welche wir otosklerotische nennen, stellen sich dar als spongiöse oder kompakte Knochenherde, denen die knorpelhaltigen Interglobularräume und meist auch der lamelläre Aufbau der normalen Labyrinthkapsel fehlen. " Oft färben sie sich stärker mit Hämatoxylin, zeigen zahlreichere Knochenzellen mit guter Kernfärbung, lebhafteren Knochenan- und abbau und größeren Gefäßreichtum als ihre Umgebung.

Bei Neugeborenen und ganz jugendlichen Individuen ist Otosklerose noch nicht angetroffen worden (Manasse) ${ }^{1}$ ). Bei einem 5jährigen, bis zur letalen Erkrankung normal hörenden Mädchen fand Siebenmann ${ }^{2}$ ) beiderseits im vorderen Fensterrahmen je einen Herd von ,progressiver Spongiosierung" und außerdem, als das seiner Ansicht nach früheste Stadium des Leidens, welches bisher histologisch beobachtet wurde, in der vorderen Wand des inneren Gehörgangs linkerseits einen weiteren, der fast ausschließlich Abbau des Kapselknochens, Fasermark und Osteoklasten in lakunär erweiterten Kanälen aufwies, während neugebildete Spongiosa sozusagen überall fehlte. Während Siebenmann ${ }^{2}$ ) und and $:$ :

$\left.{ }^{1}\right)$ Manasse, Die Ostitis chron. metaplast. der menschlichen Labyrinthkapsel (Otosklerose, Stapesankylose, Spongiosierung der Labyrinthkapsel). Wiesbaden, J. F. Bergmann 1912.

2) Siebenmann, Verh. D. otol. Ges. 1912, Hannover. 
in der Resorption des Kapselknochens das primäre sehen, glaubt Manasse ${ }^{1}$, daß in präformierten Gefäßräumen neugebildeter Knochen die normale Labyrinthkapsel. zumeist einfach verdränge, um selbst sekundär durch spongiösen und endlich kompakten Knochen ersetzt zu werden.

Während wir so bezüglich des histologischen Werdegangs der Erkrankung einstweilen auf widersprechende Hypothesen angewiesen sind, können als gesicherte Forschungsergebnisse ihre exquisite Heredität, ihre häufige Vergesellschaftung mit Erkrankungen des Labyrinths und das ïberwiegende Befallensein der Gegend zwischen Processus cochleariformis und vorderem Rand des ovalen Fensters betrachtet werden.

Die auffallende Bevorzugung dieses Teiles der Labyrinthkapsel versucht $B r u ̈ h l^{2}$ ) damit zu erklären, daß durch das Zusammentreffen von Knochen, Knorpel, Sehnenund Bindegewebe an einer Stelle, an der ausnahmsweise wie sonst nirgends im Felsenbein irritative Reize vorhanden sind, eine anatomische Prädisposition für Knochenneubildung geschaffen wird. Mayer ${ }^{3}$ ) dagegen ist auf Grund der typischen Lokalisation und Symmetrie der Herde zur Überzeugung gekommen, daß sie den Endgebieten der Arteriae nutritiae des Pyramidenknochens entsprechen und durch lokale Zirkulationsstörungen in ihnen hervorgerufen werden.

Siebenmann ${ }^{4}$ ) betont das Zusammenfallen des Locus praedilectionis mit jener Stelle der Labyrinthkapsel, an welcher besonders reichliche Knorpelreste in Gestalt von Interglobularräumen zu finden sind. Als Unikum erwähnt er bei dieser Gelegenheit ein fast hanfkorngroßes Stück hyalinen unverkalkten Knorpels in der Labyrinthkapsel einer Pfründnerin. Solche Knorpelreste, die von denen der Interglobularräume verschieden sind, haben auch Manasse ${ }^{5}$ ) und andere vereinzelt beobachtet; immerhin scheinen sie für selten an-

1) Manasse, Die Otitis chron. metaplast. der menschlichen Labyrinthkapsel (Otosklerose, Stapesankylose, Spongiosierung der Labyrinthkapsel). Wiesbaden J. F. Bergmann 1912 .

2) Brühl, Histol. Unters. der Felsenbeine eines zu Lebzeiten diagnostizierten Falles von beiderseitiger knöcherner Stapesankylose (= Otosklerose). Monatsschr. f. Ohrenheilk. 1912, S. 1289.

3) Mayer, Zur Pathogenese und Ätiologie der Otosklerose. Monatsschr. f. Ohrenheilk. 1911, s. 421.

4) Siebenmann, Multiple Spongiosierung der Labyrinthkapsel a.ls Sektionsbefund bei einem. Fall von progress. Schwerhörigkeit. Zeitschr. f. Ohrenheilk. XXXIV., S. 356.

5) Manasse, Utber knorpelhalt. Interglobularräume in der menschlichen Labyrinthkapsel. Zeitschr. f. Ohrenheilk. XXXI, S. 1. 
gesehen zu werden und einer besonderen Aufmerksamkeit nicht gewürdigt worden zu sein.

Obwohl bereits $v$. Tröltsch ${ }^{1}$ ) vermutete, daß in schwerhörigen Familien eine gewisse Gleichheit im Baue des knöchernen Ohres, insbesondere auch hinsichtlich der Fensternischen, vererbt würde, sind meines Wissens Befunde, welche als Zeichen einer örtlichen Disposition zur Bildung otosklerotischer Herde gedeutet wurden, bisher nicht erhoben worden; und die auf ihr Vorkommen in Fällen von kongenitaler Taubheit gegründete These Alexandersis), daß mindestens ihre Anlage kongenital sei, ist einstweilen ohne weitere. Stützen geblieben.

Vor kurzem nun entdeckte ich in mehreren Präparaten, darunter in den beiden Schläfenbeinen eines Individuums, zwischen Tensorsehne und ovalem Fenster größere und kleinere Reste der primären knorpligen Labyrinthkapsel, häufig durch einen von Knorpelzellen - umgebenen Bindegewebsstrang mit dem Endost am Dach der basalen Scala vestibuli oder in der Cysterna perilymphatica nahe dem vorderen Rande des ovalen Fensters verbunden. Begrenzt sind sie teils von dem lamellären Knochen der Labyrinthkapsel, teils von Interglobularräumen, in denen sich zahlreiche, von einer meist intensiv mit Hämatoxylin färbbaren Knochenhülle umgebene Gefäße verästeln. Der Knorpel selbst ist gefäßlos (vgl. Abbild. $4,5,6)$.

Die Durchsicht älterer Serien ließ mich noch mehrfach den gleichen Befund erheben und die Untersuchung einiger foetaler Schläfenbeine machte es $\mathrm{mir}$ wahrscheinlich, daß diesen Knorpelresten entsprechend im Promontorium die knorplige Kapsel am spätesten durch Knochen ersetzt wird; daß ferner der erwähnte Bindegewebsstrang beim Foetus der letzten Monate zum mindesten ziemlich regelmäßig zu finden ist. ${ }^{3}$ ) (s. Abb. 1, 2, 3).

Der Schluß, daß größere Reste hyalinen Knorpels an dieser Stelle bei Entwicklungshemmung verschiedener Ätiologie häufiger als anderswo in der Labyrinthkapsel erhalten bleiben, scheint danach nicht ungerechtfertigt, die Annahme, daß wir

1) v. Tröltsch, Lehrbuch der Ohrenheilkunde, 5. Aufl., 1873.

2) Alexander, Das Gehörorgan der Kretinen. Arch. f. Ohrenheilkunde LXXVIII., \$. 54.

$\left.{ }^{3}\right)$ Vgl. z. B. auch Abbildung 9 auf $\mathrm{T}, \mathrm{X}$ zu Hofer, Beitr. zur path. Anat. des Ohres bei kongenitaler Syphilis. A. f. O. 1912. XC. S. 117. 
in ihnen einen Locus minoris resistentiae der otosklerotischen Erkrankung gegenüber, vielleicht sogar ein Vorstadium des otosklerotischen Herdes "zu sehen haben, nicht fernliegend und sehr verführerisch.

Nicht nur würde durch die Lage der Knorpelreste, - welche der Feststellung Politzersis), daB die typische Otosklerose eine primäre Erkrankung der Labyrinthkapsel darstellt, entspricht, - die Symmetrie und die Häufigkeit der Herde gerade an dieser Stelle besser erklärt werden als durch die Hypothesen Brühls und Mayers, auch die Heredität der Erkrankung und der von Habermann ${ }^{2}$ ), Gradenigo $o^{3}$ ) und anderen behauptete Zusammenhang eines Teils der Otosklerosefälle mit kongenitaler Lues wäre damit dem Verständnis näher gerückt.

Daß kongenitale Lues häufig eine Verzögerung der Ossifikation in der Labyrinthkapsel zur Folge hat, ist durch Asais ${ }^{4}$ ) und Hofers ${ }^{5}$ ) Arbeiten erwiesen. Ähnliche Verhältnisse dürften auch beim Kretinismus und anderen Konstitutionsanomalien vorliegen, die als Ursache der Otosklerose in Frage kommen.

Die Beobachtung, daß die Disposition zur Bildung otosklerotischer Herde und mangelhafte Entwicklung oder Schwäche der nervösen Elemente des inneren Ohres sich nicht selten im Individuum und in der Familie zusammenfinden, würde unserer Vermutung, daß erstere durch ungenügende Ossifikation der Labyrinthkapsel gegeben wird, nicht entgegenstehen.

Das auffällige Überwiegen des weiblichen Geschlechtes unter den Kranken spricht nach Denker ${ }^{6}$ ) geradezu für schädliche Einflüsse, die sich während des Foetallebens geltend machen, als Ursache der Otosklerose, da ihnen gegenüber nachweisbar weibliche Individuen weniger widerstandsfähig sind als männliche. Von 5 Fällen mit Knorpelresten,

1) Politzer, Ưber primäre Erkrankung der knöchernen Labyrinthkapsel. Zeitschr. f. Ohrenheilk. XXV., S. 309.

$\left.{ }^{2}\right)$ Habermann, Zur Patholog. der sogen. Otosklerose. Archiv f. Ohrenheilkunde LX., S. 37 .

$\left.{ }^{3}\right)$ Gradenigo, 85. Vers. D. Naturforscher u. Ärzte. Wien 1913. Ref. Zbl. f. Ohrenheilkunde XI.; S. 524 .

4) Asai, Beitr. z. path. Anat. des Ohres bei Lues hereditaria. J. F. Bergmann, Wiesbaden 1908.

5) Hofer, Beitr. zur path. Anat. des Ohres bei kongenitaler Syphilis. Archiv f. Ohrenheilkunde XO, S. 117.

6) Denker, Die Otosklerose. Wiesbaden 1904. 
in denen das Geschlecht mir bekannt ist, betreffen vier Mädchen und Frauen.

Zur Erörterung der Frage, in welcher Weise der Übergang vom Knorpel zum otosklerotischen Knochen zu denken sei, ob durch primäre Auflösung des Knorpels und Knochenneubildung von den GefäBschlingen der Umgebung her oder durch einfache Metaplasie und sekundäre Spongiosierung des Herdes und seiner Umgebung, fehlt mir eigenes Material. Das Einsetzen derartiger Prozesse in der Pubertät, ihr Fortschreiten in der Schwangerschaft usw. findet hinreichende Analoga in der Pathologie.

Sollte sich für die typisch verlaufende, hereditäre Otosklerose meine Annahme einer örtlichen Disposition durch mangelhafte Resorption der knorpligen Labyrinthkapsel bestätigen, so würde das für unsere interne Therapie bedeuten, daß sie nach Grunertsil) Anregung bei Angehörigen hereditär belasteter Familien viel frïher als die Symptome, am besten schon im Foetalleben einsetzen müßte, um ihre Erfolge zu verbessern.

Zum Schluß sei es mir gestattet, Herrn Professor Ricker, Magdeburg, für die freundliche Überlassung: des Materials meinen herzlichsten Dank zu sagen.

\section{Befunde.}

1. Foetus I (ca. 7. Monat).

a.) Schnitt durch Macula utriculi, Pars vestibularis Cochleae, Nische zum runden Fenster: Der quergetroffene Facialis liegt, von der Dura gedeckt, in die obere Fläche der Labyrinthkapsel eingebettet. Von seiner Scheide aus erstreckt sich nach unten und paukenwärts ein Stitck zellreichen hyalinen Knorpels in den weiten, nur von spärlichen Spongiosabälkchen durchzogenen Markraum der enchondral gebildeten Labyrinthkapsel. Labyrinthwärts ist ihm neugebildeter Knochen angelagert, paukenwärts sind seine Zellen gequollen, in Teilung und Auflösung, stellenweise, nach der Tinktion za urteilen, in Verkalkung begriffen. Mit seinem unteren Ende nähert sich der beschriebene Knorpelherd einem zweiten, der dem Rande des ovalen Fensters angehört.

b) Schnitt durch Schnecke, inneren Gehörgang bzw. Facialiskanal, Proc. cochleariformis, Tensorsehne, Hammergriff, Ambo B. Zwischen Facialisknie und mittlerer Schneckenwindung liegt ein gröBerer, makroskopisch erkennbarer Knorpelrest, der einexseits bis an die Nervenscheide heranreicht, andererseits nach dem weiten

1) Grunert, Über die Ergebnisse in der allgemein pathol. $u_{*}$ path. anat. Forschung des kranken Mittelohres usw. Archiv für Ohrenheilkunde LX., S. 124.

Archiv f. Ohrenheilkunde. Bd. 95. 
Markraum der enchondral gebildeten Labyrinthkapsel zu in Auflösung begriffen ist.

2. Foetus II (ca. 8. Monat).

a) Schnitt durch Hammergriff und Tensorsehne, Proc. cochleariformis, M. stapedius, Stapes, Macula sacculi und Schnecke: Zwischen mittlerer Schneckenwindung, Proc. cochleariformis und Stapesnische liegt ein größerer, fast völlig von neugebildetem Knochen umlagerter Herd hyalinen Knorpels, der durch einen gefäßführenden, größtenteils von Knorpel umgebenen Bindegewebsstrang sowohl mit der Stapesnische (Fig. 1) wie mit dem Endost der Cysterna perilymphatica (Fig. 2) in Verbindung steht.

b) Schnitt durch Proc. brevis, Hammerkopf und -griff, Tensorsehne, Schneckenbasis und mittlere Schneckenwindung (Fig. 3): Zwischen Proc. cochleariformis und Dach der unteren Schneckenwindung findet sich ein ca. $1^{1 / 2} \mathrm{~mm}$ langer Knorpelrest, dessen mittlerer Teil am Rande die Zeichen fortschreitender Resorption zeigt, während er oben und unten, von einer Art Kapsel umhüllt, sich scharf gegen die benachbarten Knochenbälkchen und Markräume abgrenzt. Nach dem ovalen Fenster zu setzt sich der Knorpelherd in eine schmale, von Knorpel umgebene bindegewebige Ausstrahlung des Ligamentum annulare fort. Im übrigen findet sich Knorpel am Promontorium nur in den Interglobularräumen der Spongiosabälkchen.

3. Frühgeburt, Mikrotie (auf der andern Seite mit Atresie des Gehörgangs ).

Schnitt durch Schneckenbasis und inneren Gehörgang: Oberhalb der basalen Schneckenwindung, unmittelbar vor dem vorderen Rand des ovalen Fensters liegt teils von lamellärem Knochen, teils von Mark umgeben, ein Stück hyalinen, zellreichen Knorpels, dessen labyrinthwärts gelegener Rand Kalkeinlagerungen aufweist.

4. Säugling, Lues congenita. Otitis media acuta.

Dicht vor dem vorderen Rande des ovalen Fensters entspringt vom Endost der Cysterna perilymphatica ein von Knorpel umgebener schmaler Bindegewebsstrang, der an einem kleinen Herde hyalinen Knorpels in Nähe des Proc. cochleariformis endet.

5. W.R., 1\% $1 / 2$ jähr. Mäd chen, Todan Miliartuberkulose. Beiderseits Otitis media acuta.

a) Zwischen Facialiskanal und Proc. cochleariformis einer-, Schneckenbasis andererseits liegt ein ca. $2 \mathrm{~mm}$ langes Stück zellreichen hyalinen Knorpels. Begrenzt wird es von Lymphspalten und Interglobularräumen, in denen sich zahlreiche von stark tingierten Knochenhüllen umgebene Gefäße verzweigen.

b) An der gleichen Stelle wie im Schläfenbein der anderen Seite finden sich auch hier Knorpelreste, von denen ein Bindegewebsstrang zum Endost am Dache der Scala vestibuli nahe ihrem Utbergang in den Vorhof verläuft.

6. 10 jähr. Mädchen, Tod an Scarlatina. Otitis media acuta.

Unterhalb des Facialisknies zwischen Proc. cochleariformis, mittlerer Schneckenwindung und Macula utriculi liegt teils von lamellär gebautem kompaktem Knochen, teils von Interglobularräumen umschlossen, ein tief mit Hämatoxylin tingiertes Stück hyalinen Knorpels (Fig. 4), das sich bis in die Höhe des ovalen Fensters hinaberstreckt, von dessen vorderem unterem Rande ein knorpelamgebener Bindegewebsstrang sich ihm nähert. Dem. Ligamentum annulare ist vorn unten ein kleines Stück Knorpel eingelagert. 
7. Gr., 16 jähr. Le hrling. Tod an Hirnabszeß nach Cholesteatomeiterung (Fig. 5. u. 6).

Meist von großen Interglobularräumen, in denen sich zahlreiche, von dunkelgefärbtem Knochen umgebene Gefäße verästeln, stellenweise auch von lamellärem Knochen begrenzt, liegt zwischen Stapesnische und Schnecke ein schon makroskopisch deutlich erkennbares Stück tief mit Hämatoxylin gefärbten Knorpels, welches bis an das Periost der Stapesnische heranreicht. Nach unten findet sich in seiner Verlängerung ein schmaler Strang Bindegewebe, der, von Knorpelzellen umgeben, zum Teil verkalkt, nach dem Endost der Cysterna perilymphatica in Nähe des vorderen unteren Fensterrandes zieht.

\section{Frau, Tod an Meningitis.}

Schnitt durch Proc. cochleariformis, untere Schneckenwindung und tlach durch die Macula sacculi: Nahe dem Proc. cochleariformis erblickt man ein tiefdunkel gefärbtes, großenteils von lamellärem Knochen, im übrigen von Interglobularräumen begrenztes Stück Knorpel, mehr labyrinthwärts den Querschnitt eines von Knorpel eingeschlossenen Bindegewebsstranges, der sich weiterhin zum Endost am Dach der Scala vestibuli verfolgen läßt.

9. Frau, Fibrosarkommetastase im Bulbus ven. jugular.

Vertikalschnitt durch die Schneckenbasis an der Mündung des Aquaeduktus Cochleae: Dicht oberhalb der Scala vestibuli findet sich von Interglobularräumen umgeben ein kleines Stück kompakten. hyalinen Knorpels.

Vorstehende Arbeit wurde Mitte März bei der Redaktion eingeliefert. Inzwischen hat Herr Professor Manasse in Bd. 95 S. 145 über ähnliche Beobachtungen berichtet. In aller Kürze möchte ich mir erlauben, auffolgende Unterschiede meiner Befunde von den seinen hinzuweisen: In keinem meiner Fälle habe ich auch bei nochmaliger Durchsicht der Präparate besonders auffallende Herde von Fettmark oder wesentlich von dem übrigen, normalen Knochen verschieden gefärbte Knochengebilde in Nähe der Knorpelreste gefunden. - In keinem Fall finden sich atrophiseh-degenerative Veränderungen des inneren Ohres. - In Fall 3. fehlte die Knorpelinsel auf dem zweiten, atretischen, Ohr. - Der Knochen unmittelbar um die Gefäße ist in der Umgebung der Knorpelherde oft stärker mit Hämatoxylin gefärbt als an den Gefäßen dor übrigen Labyrinthwand. - Der äußerste Rand der Knorpelinseln hat sich stellenweise mit Eosin gefärbt und hebt sich dadurch kapselartig von dem angrenzenden Knorpel der Interglobularräume ab. - Ein direkter oder indirekter $\mathrm{Zu}$ - 
246 W. SCHOETZ, Gibt es eine kongenitale örtliche Disposition usw.

sammenhang der Knorpelreste mit dem Knorpelbelag des Fensterrahmens oder dem Endost ist in der Mehrzahl meiner Fälle nachzuweisen und scheint beim Fötus die Regel zu bilden.

\author{
Zeichenerklärungen $\mathrm{zu}$ den Figuren 1-6. \\ St. = Stapes. \\ T. = Tensor. \\ K. = Knorpel. \\ C. = Cochlea. \\ C. $m . \mathrm{W} .=$ Mittlere Schnockenwindung. \\ C. B. = Basale Schneckenwindung. \\ M. S. = Macula sacculi.
}



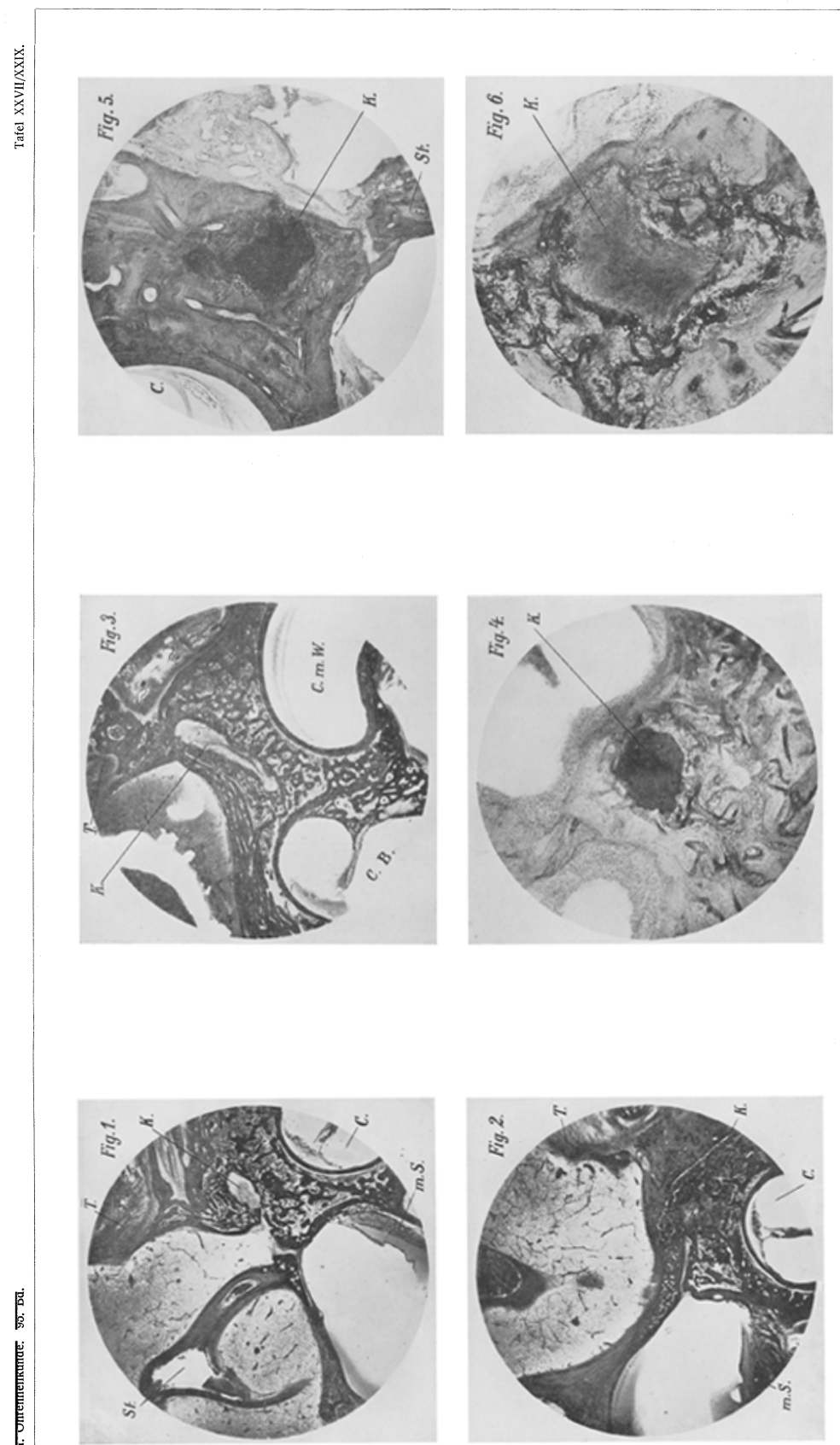